\title{
Serum adiponectin in HIV-1 and hepatitis C virus mono- and co- infected Kenyan injection drug users
}

\author{
Eric M Ndombi ${ }^{1}$, Valentine Budambula², Mark K Webale ${ }^{3}$, Francis 0 Musumba ${ }^{3}$, \\ Jesca 0 Wesongah ${ }^{4}$, Erick Mibei ${ }^{5}$, Aabid A Ahmed, Raphael Lihana ${ }^{6}$ and Tom Were ${ }^{7}$ \\ Bomu Hospital, Mombasa, Kenya \\ ${ }^{1}$ Department of Pathology, Kenyatta University, Nairobi, Kenya \\ ${ }^{2}$ Department of Environment and Health Sciences, Technical University of Mombasa, Mombasa, Kenya \\ ${ }^{3}$ Department of Biomedical Sciences and Technology, Maseno University, Maseno, Kenya \\ ${ }^{4}$ Department of Medical Laboratory Sciences, Jomo Kenyatta University of Agriculture and Technology, Juja, Kenya \\ ${ }^{5}$ Kapkatet Campus, University of Kabianga, Kericho, Kenya \\ ${ }^{6}$ Centre for Virus Research, Kenya Medical Research Institute, Nairobi, Kenya \\ ${ }^{7}$ Department of Medical Laboratory Sciences, Masinde Muliro University of Science and Technology, \\ Post Box 190-50100 Kakamega, Kenya
}

\author{
Correspondence \\ should be addressed \\ to T Were \\ Email \\ mugogwe@yahoo.com
}

\begin{abstract}
Adiponectin is an important marker of anthropometric profiles of adipose tissue. However, association of adiponectin and adiposity in HIV mono- and co-infected and hepatitis (HCV) injection drug users (IDUs) has not been elucidated. Therefore, the relationship of total adiponectin levels with anthropometric indices of adiposity was examined in HIV monoinfected (anti-retroviral treatment, ART-naive, $n=16$ and -experienced, $n=34$ ); HCV monoinfected, $n=36$; HIV and HCV co-infected (ART-naive, $n=5$ and -experienced, $n=13$ ); uninfected, $n=19$ IDUs; and healthy controls, $n=16$ from coastal Kenya. Anthropometric indices of adiposity were recorded and total circulating adiponectin levels were measured in serum samples using enzyme-linked immunosorbent assay. Adiponectin levels differed significantly amongst the study groups $(P<0.0001)$. Post-hoc analyses revealed decreased levels in HIV mono-infected ART-naive IDUs in comparison to uninfected IDUs $(P<0.05)$ and healthy controls $(P<0.05)$. However, adiponectin levels were elevated in HCV mono-infected IDUs relative to HIV mono-infected ART-naive $(P<0.001)$ and -experienced $(P<0.001)$ as well as HIV and HCV co-infected ART-naive $(P<0.05)$ IDUs. Furthermore, adiponectin correlated with weight ( $\rho=0.687 ; P=0.003)$ and $\operatorname{BMI}(\rho=0.598 ; P=0.014)$ in HIV mono-infected ART-naive IDUs; waist circumference $(\rho=-0.626 ; P<0.0001)$, hip $(\rho=-0.561 ; P=0.001)$ circumference, and bust-to-waist ratio $(\rho=0.561 ; P=0.001)$ in HIV mono-infected ARTexperienced IDUs; waist girth $(\rho=0.375 ; P=0.024)$ in HCV mono-infected IDUs; and waistto-hip ratio ( $\rho=-0.872 ; P=0.048)$ in HIV and HCV co-infected ART-naive IDUs. Altogether, these results suggest suppression of adiponectin production in treatment-naive HIV monoinfected IDUs and that circulating adiponectin is a useful surrogate marker of altered adiposity in treatment-naive and -experienced HIV and HCV mono- and co-infected IDUs.
\end{abstract}
Key Words
- adiponectin
- anthropometric markers of adiposity
- hepatitis $C$ virus
- HIV
- anti-retroviral treatment
- injection drug users

Endocrine Connections (2015) 4, 223-232

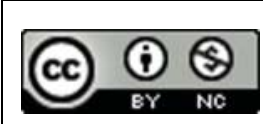

This work is licensed under a Creative Commons Attribution-NonCommercial 4.0 International License. 


\section{Introduction}

Injection drug use and associated co-morbidities are an increasing public health burden in the world (1). An estimated 15.9 million people worldwide are injection drug users (IDUs), comprising 3 and 10 million HIV and hepatitis $\mathrm{C}$ virus $(\mathrm{HCV})$ infections respectively $(2,3)$. Due to overlaps in risks and modes of acquiring infection, $75 \%$ of the global injection drug using population is co-infected with HIV and HCV viruses (4). The interaction of HIV and HCV infections and injection drug use is largely undefined. However, injection drugs such as heroin promotes disease progression in HIV and HCV infected individuals $(5,6)$.

Despite anti-retroviral treatment improving quality of life, adverse metabolic syndromes frequently develop in HIV infected individuals. For instance, accumulation of visceral and dorso-cervical adipose tissue, breast enlargement, and loss of facial and limb subcutaneous fat are attributable to anti-retroviral drugs, especially nucleoside reverse transcriptase inhibitors (NRTI) and protease inhibitors (PI) $(7,8)$. In addition, HIV and HCV monoand co-infected individuals are at an increased risk of developing lipodystrophy $(7,9,10)$, suggesting propensity for adipose redistribution and dysfunction. Although anthropometric indices correlate with total body, central body, subcutaneous and visceral adiposities $(11,12)$, surrogate markers of adiposity in IDUs are largely undefined. However, previous studies showing higher rates of malnutrition (BMI $<18.5 \mathrm{~kg} / \mathrm{m}^{2}$ ), lower body weight and fat mass in HIV-infected and uninfected IDUs $(13,14)$, indicate that anthropometric measures are valuable in estimating adiposity in IDUs. In addition, the link between lipodystrophy and adipocyte dysfunction (15) suggests that dysregulation in circulating adipokine production correlates with adiposity.

Adiponectin is an adipocytokine produced almost exclusively by adipose tissue with pleiotropic regulatory effects on carbohydrate, lipid and protein metabolism (16). Previous studies indicated reduced circulating adiponectin levels in heroin and crack cocaine addicts along with cigarette smokers $(17,18,19)$. In contrast, chronic alcohol consumption is associated with increased serum adiponectin levels (20), suggesting that substance consumption promotes alterations in the production of circulating adiponectin. In addition, previous studies indicated decreased circulating adiponectin levels in lipodystrophic ART-naive and -experienced HIV patients $(21,22,23)$. Likewise, decreased serum adiponectin levels are associated with higher BMI in HCV patients (24), indicating that adiponectin is an important correlate of adiposity in HIV and HCV infections.

Although injection drug use alters adiponectin and anthropometric profiles of HIV infected and -uninfected IDUs $(17,25)$, host markers underlying adipose tissue status in IDUs presenting with HIV and HCV mono- and co-infections are largely unknown. We have previously shown altered clinical chemistry profiles in HIV infected ART-naive and -experienced injection heroin users from coastal Kenya (26). In the present study, we determined serum total adiponectin levels and their association with anthropometric markers among HIV and HCV mono- and co-infected ART-naive and -experienced IDUs.

\section{Materials and methods}

\section{Study site and population}

This hospital-based, cross-sectional study was conducted as part of a wider study investigating the nutritional and microbiological correlates of HIV infection among IDUs. Upon enrolment, the study participants were stratified as follows: i) HIV mono-infected ART-naive; ii) HIV monoinfected ART-experienced; iii) HCV mono-infected; iv) HIV and HCV co-infected ART-naive; v) HIV and HCV co-infected ART-experienced; and vi) HIV and HCV negative (uninfected) IDUs; and vii) healthy controls. Injection drug users were defined as individuals with a history of injection drug use, reporting injecting any illicit drugs at least once in the previous month, and showing evidence of injection needle-stick scars, while healthy controls were individuals reporting having never used any illicit drugs from the UNODC report (1). At the time of enrolment in the study, ART-naive study participants had not been initiated on ART while individuals on ART (ARTexperienced) were receiving first line anti-retroviral drugs. These comprised of tenofovir (TDF) or zidovudine (AZT) + lamivudine $(3 \mathrm{TC})+$ nevirapine (NVP) or efavirenz (EFV) (27). The protease inhibitors were mainly used in postexposure prophylaxis and second-line HIV treatment or and as part of the TDF or abacavir $(\mathrm{ABC})+3 \mathrm{TC}+$ lopinavirritonavir (LPVr) regimen (27). Individuals with underlying disease conditions, including diabetes mellitus, were excluded from the study. Approximately, $10 \mathrm{ml}$ of venous blood samples were collected from the study participants and aliquoted into plain and EDTA BD vacutainer tubes (BD, Franklin Lakes, NJ, USA). EDTA blood samples were used for $\mathrm{CD}^{4+} \mathrm{T}$ cell enumeration and plasma preparation

This work is licensed under a Creative Commons Attribution-NonCommercial 4.0 International License. 
for HIV and HCV sero-testing and HIV viral load quantification as previously described (26). All blood samples were collected in the morning before $01000 \mathrm{~h}$ and haematologic analyses were performed within $30 \mathrm{~min}$ of blood collection to minimize variability in the measurements. Serum samples were prepared from plain tube blood and frozen at $-80^{\circ} \mathrm{C}$ until used for batched measurements of adiponectin.

\section{Anthropometric assessment}

Anthropometric measures of the study participants were obtained according to WHO criteria (28). Height and weight were measured while participants stood upright, with no shoes, and wearing light clothing. Waist circumference was measured at the midpoint of the lower margin of the last palpable rib and the top of the iliac crest (hip bone). Hip circumference was obtained around the maximum circumference of the buttocks. Bust circumference was taken around the chest and below the armpits after exhalation while mid-upper-arm circumference (MUAC) was measured at the mid-point between the tip of the shoulder and the tip of the elbow. Height (m) and weight $(\mathrm{kg})$ measurements were used to calculate BMI (weight/height, $\mathrm{kg} / \mathrm{m}^{2}$ ). Waist-to-hip ratio and bust-to-hip ratio were calculated by dividing waist circumference $(\mathrm{cm})$ with hip circumference $(\mathrm{cm})$ and bust circumference $(\mathrm{cm})$ by hip circumference $(\mathrm{cm})$ respectively.

\section{Total adiponectin enzyme-linked immunosorbent assay}

Total adiponectin concentrations were determined in serum using commercial ELISA reagents according to manufacturer's protocols (R\&D Systems, Minneapolis, MN, USA) with modifications. Briefly, ELISA microtitre plate wells (MaxiSorp, Denmark) were coated with $100 \mu \mathrm{l}$ of $2.0 \mu \mathrm{g} / \mathrm{ml}$ mouse anti-human adiponectin monoclonal antibody. The wells were aspirated and washed two times using $300 \mu \mathrm{l}$ of wash buffer (PBS $+0.05 \%$ Tween-20, pH 7.2) and blocked with $100 \mu$ l of blocking buffer (1\% BSA, BSA+ PBS, pH 7.2). The wells were washed four times with wash buffer and $50 \mu$ l of diluted standards, and samples were added to respective wells in duplicate, then incubated with shaking for $2 \mathrm{~h}$ at room temperature. About $100 \mu \mathrm{l}$ of $2.0 \mu \mathrm{g} / \mathrm{ml}$ biotinylated detection mouse anti-human adiponectin antibody were added to each well and incubated for $2 \mathrm{~h}$ at room temperature. Streptavidin-horse radish peroxidise was added at $50 \mu \mathrm{l} /$ well and incubated for $20 \mathrm{~min}$ at room temperature. After four washes with wash buffer, $100 \mu \mathrm{l}$ of tetramethylbenzinidine substrate were added to the wells and the plates were incubated in the dark for $20 \mathrm{~min}$ at room temperature. Colour development was stopped by addition of $1 \mathrm{~N} \mathrm{H}_{2} \mathrm{SO}_{4}$ and optical densities of each well were read at $450 \mathrm{~nm}$ with a reference of $630 \mathrm{~nm}$ using the Dynatech MR5000 Microplate Reader (Dynex Technologies, Inc., Chantilly, VA, USA). Sample concentrations were calculated using the appropriate standard calibration curves of the corresponding recombinant human adiponectin protein included in each assay plate.

\section{Ethical considerations}

The study was approved by Kenyatta University Ethical Review Committee. All study participants provided written informed consent prior to enrolment. Confidentiality of the study participant's information was ensured throughout the study. All the study participants were provided with free health education on sexually transmitted infections including HIV, hepatitis B and C, tuberculosis, hygiene, and nutrition. Participants testing positive for HIV and hepatitis virus infections were referred to the comprehensive care centres at Bomu Hospital or the Coast General Referral Hospital for treatment, care, and support.

\section{Data analysis}

Statistical analysis was conducted using GraphPad Prism Software v5 (GraphPad, Inc., San Diego, CA, USA). Nutritional and laboratory measures were summarized as medians (interquartile range, IQR) while gender, malnutrition and substance use summarized as proportions were tabulated. Adiponectin levels across study groups were presented as box plots. Statistical comparisons in categorical data across study groups were performed using $\chi^{2}$-test as appropriate. Comparisons in continuous data across study groups were performed using Kruskal-Wallis test followed by Dunn's correction for multiple comparisons. Spearman's rank correlation tests were used to examine the associations of adiponectin levels with nutritional measures. All tests were two-tailed with statistical significance set at an $\alpha$-value of $5 \%$.

\section{Results}

A total of 139 adults (males, $n=90$ and females, $n=49$ ) were recruited into the study. The study participants comprised of IDUs categorised into HIV mono-infected (ART-naive, $n=16$ and -experienced, $n=34$ ) HCV monoinfected $(n=36)$; HIV and HCV co-infected (ART-naive,

This work is licensed under a Creative Commons Attribution-NonCommercial 4.0 International License. 
$n=5$ and -experienced, $n=13)$; uninfected ( $n=19)$ individuals; and healthy controls $(n=16)$. Demographic and clinical characteristics of the study participants are summarised in Table 1. Age of the study participants differed significantly amongst the study groups $(P=0.001)$ and was higher in the HCV mono-infected group compared to healthy individuals $(P<0.05)$. The frequencies of female gender in the study groups were 50.0, 61.8, $2.8,20.0,15.4,42.1$, and 50.0\% respectively.

Although most of the nutritional measures (height, weight, BMI, waist circumference, bust circumference, hip circumference, and MUAC) were significantly different across the study groups $(P<0.01)$, waist-to-hip ratio $(P=0.290)$ and bust-to-waist ratio $(P=0.052)$ were not significantly different across the groups. Post-hoc analysis showed significantly higher height in HCV mono-infected
IDUs (median, 1.72; IQR, $0.07 \mathrm{~m}$ ) compared to healthy controls (median, 1.64; IQR, $0.14 \mathrm{~m} ; P<0.05$ ). In contrast, weight was lower in the $\mathrm{HCV}$ mono-infected IDUs (median, 55.0, IQR, $9.5 \mathrm{~kg}$ ) relative to healthy controls (median, 62.0; IQR, $18.0 \mathrm{~kg} ; P<0.05$ ). Likewise, BMI was lower in HIV mono-infected ART-experienced (median, 17.99; IQR, $\left.2.10 \mathrm{~kg} / \mathrm{m}^{2} ; P<0.05\right)$, HCV mono-infected (median, 18.44; IQR, $2.89 \mathrm{~kg} / \mathrm{m}^{2} ; P<0.05$ ), and uninfected (median, 18.69, IQR, $2.84 \mathrm{~kg} / \mathrm{m}^{2} ; P<0.05$ ) IDUs compared to healthy controls (median, 22.84; IQR, $6.01 \mathrm{~kg} / \mathrm{m}^{2}$ ). Likewise, waist circumference was lower in the co-infected ART-experienced (median, 77.0; IQR, $13.0 \mathrm{~cm} ; P<0.05$ ) and -naive (median, 74.0; IQR, $13.0 \mathrm{~cm} ; P<0.05$ ) IDUs compared to healthy controls (median, 86.0; IQR, $12.0 \mathrm{~cm})$. Bust girth was also lower in co-infected ART-experienced and HCV mono-infected (median, 84.0;

Table 1 Baseline demographic and laboratory characteristics of the study participants.

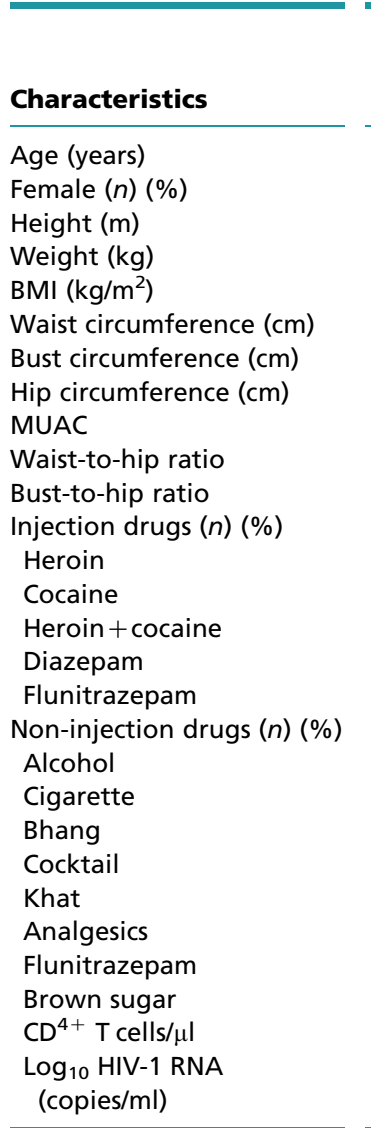

\begin{tabular}{|c|}
\hline $\begin{array}{c}\text { Healthy } \\
\text { controls } \\
(n=16)\end{array}$ \\
\hline $26.0(8.5)$ \\
\hline $8(50.0)$ \\
\hline $1.64(0.14)$ \\
\hline $62.0(18.0)$ \\
\hline $22.84(6.01)$ \\
\hline $86.0(12.0)$ \\
\hline $89.5(11.0)$ \\
\hline $100.0(16.5)$ \\
\hline $29.0(7.0)$ \\
\hline $0.88(0.11)$ \\
\hline $1.06(0.15)$ \\
\hline
\end{tabular}

\begin{tabular}{ccc}
$\begin{array}{c}\text { HIV }- \text { HCV }- \\
(n=19)\end{array}$ & & $\begin{array}{c}\text { HIV }+ \text { HCV }+ \\
\text { ART }+ \\
(n=13)\end{array}$ \\
\cline { 1 - 1 } $\begin{array}{cl}27.7(7.8) \\
8(42.1)\end{array}$ & & $\begin{array}{c}32.4(7.3) \\
2(15.4)\end{array}$ \\
$1.72(0.17)$ & & $1.71(0.14)$ \\
$54.0(9.0)$ & & $54.0(8.5)$ \\
$18.69(2.84)^{a}$ & & $18.36(2.81)$ \\
$76.0(6.0)$ & & $77.0(13.0)^{a}$ \\
$88.0(7.0)$ & & $85.0(12.0)^{a}$ \\
$90.0(7.0)$ & & $91.0(13.5)^{a}$ \\
$25.0(4.0)^{a}$ & & $26.1(2.0)$ \\
$0.84(0.07)$ & & $0.84(0.07)$ \\
$1.14(0.09)$ & & $1.10(0.06)$
\end{tabular}

\begin{tabular}{c} 
HIV + HCV + \\
ART $-(n=5)$ \\
\hline $34.0(8.1)$ \\
$1(20.0)$ \\
$1.60(0.20)$ \\
$50.0(19.0)$ \\
$18.69(2.85)$ \\
$74.0(13.0)^{a}$ \\
$81.5(10.5)$ \\
$89.0(15.0)$ \\
$25.0(5.1)$ \\
$0.80(0.06)$ \\
$1.18(0.10)$
\end{tabular}

\begin{tabular}{|c|c|}
\hline $\begin{array}{l}\mathbf{H C V}+ \\
(n=36)\end{array}$ & $\begin{array}{l}\text { HIV }+ \\
\text { ART }+ \\
(n=34)\end{array}$ \\
\hline $33.8(12.4)^{a}$ & $31.4(6.1)$ \\
\hline $1(2.8)$ & $21(61.8)$ \\
\hline $1.72(0.07)^{a}$ & $1.67(0.10)$ \\
\hline $55.0(9.5)^{a}$ & $51.5(5.0)$ \\
\hline $18.44(2.89)^{a}$ & $17.99(2.10)$ \\
\hline $77.5(10.4)$ & $71.0(8.0)$ \\
\hline $84.0(6.0)^{a}$ & $82.5(4.4)$ \\
\hline $91.5(6.0)$ & $88.0(8.0)$ \\
\hline $26.0(3.0)^{a}$ & $24.0(2.0)^{a}$ \\
\hline $0.83(0.06)$ & $0.81(0.05)$ \\
\hline $1.10(0.13)$ & $1.15(0.13)$ \\
\hline
\end{tabular}

\begin{tabular}{|c|c|}
\hline $\begin{array}{c}\text { HIV + ART - } \\
\quad(n=16)\end{array}$ & $\boldsymbol{P}$ \\
\hline 30.7 (5.1) & 0.001 \\
\hline $8(50.0)$ & - \\
\hline $1.68(0.14)$ & 0.009 \\
\hline $54.0(6.8)$ & $<0.0001$ \\
\hline $19.47(3.67)^{a}$ & $<0.0001$ \\
\hline $72.0(5.8)$ & $<0.0001$ \\
\hline $84.0(8.3)$ & $<0.0001$ \\
\hline $90.0(7.5)$ & 0.003 \\
\hline $24.5(3.0)^{a}$ & $<0.000$ \\
\hline $0.83(0.08)$ & 0.290 \\
\hline $1.15(0.07)$ & 0.052 \\
\hline
\end{tabular}

$\begin{array}{lc}0(0.0) & 19(100.0) \\ 0(0.0) & 0(0.0) \\ 0(0.0) & 0(0.0) \\ 0(0.0) & 0(0.0) \\ 0(0.0) & 0(0.0) \\ & \\ 0(0.0) & 7(36.8) \\ 0(0.0) & 15(78.9) \\ 0(0.0) & 7(36.8) \\ 0(0.0) & 4(21.1) \\ 0(0.0) & 4(21.1) \\ 0(0.0) & 7(36.8) \\ 0(0.0) & 9(47.4) \\ 0(0.0) & 2(10.5) \\ 81(487) & 985(436) \\ - & - \\ & \end{array}$

10 (76.9)

$5(100.0)$

$31(86.1)$

$29(85.3)$

2 (15.4)

1 (7.7)

$0(0.0)$

1 (7.7)

7 (53.8)

$10(76.9)$

6 (46.2)

5 (38.5)

$4(30.8)$

2 (15.4)

7 (53.8)

2 (15.4)

473 (256)

$2.90(1.82)$

$5(100.0)$
$0(0.0)$
$0(0.0)$
$0(0.0)$
$0(0.0)$

4 (11.1)

$1(2.8)$

4 (11.1)

4 (11.8)

1 (2.9)

2 (5.9)

$0(0.0)$

\section{1 (20.0)}

$5(100.0)$

$3(60.0)$

4 (80.0)

$0(0.0)$

$0(0.0)$

$1(20.0)$

$0(0.0)$

$585(682)^{c}$

$2(5.6)$

5 (13.9)

19 (55.9)

24 (66.7)

$27(75.0)$

13 (36.1)

$9(25.0)$

8 (22.2)

23 (63.9)

3 (8.3)

4.54 (3.43)

$$
24(70.6)
$$

$20(58.8)$

16 (47.1)

$14(41.2)$

5 (14.7)

23 (67.6)

6 (17.6)

$362(503)^{a, b}$

2.59 (2.65)

15 (93.8)

1 (6.3)

$0(0.0)$

2 (12.5)

$0(0.0)$

$10(62.5)$

$11(68.8)$

8 (50.0)

6 (37.5)

5 (31.3)

7 (43.8)

$10(62.5)$

2 (12.5) $946(659)$

Data are presented as medians (interquartile range) or as indicated. HIV, human immunodeficiency virus; HCV, hepatitis C virus; ART, anti-retroviral treatment; HIV + HCV + ART +, HIV and HCV co-infected ART-experienced; HIV+HCV+ART-, HIV and HCV co-infected ART-naive; HCV +, HCV mono-infected; HIV+ART+, HIV mono-infected ART-experienced; HIV+ART-, HIV mono-infected ART-naive; HIV+HCV +, uninfected. MUAC, mid upper arm circumference; data analysis was performed using Kruskal-Wallis tests. Following the Kruskal-Wallis tests, post-hoc correction for multiple comparisons were performed using the Dunn's multiple comparison test. Significant $P$ values are shown in bold. ${ }^{\text {a }} P<0.05$ vs healthy controls; ${ }^{b} P<0.001$ vs uninfected IDUs; ${ }^{C} P<0.05$ vs HCV mono-infected group.

http://www.endocrineconnections.org DOI: 10.1530/EC-15-0071
(C) 2015 The authors Published by Bioscientifica Ltd

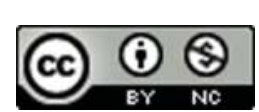

This work is licensed under a Creative Commons Attribution-NonCommercial 4.0 International License. 
IQR, $6.0 \mathrm{~cm} ; P<0.05)$ IDUs than healthy controls (median, 89.5; IQR, $11.0 \mathrm{~cm}$ ). Moreover, hip circumference was lower in co-infected ART-experienced IDUs (median, 91.0; IQR, $13.5 \mathrm{~cm} ; P<0.05$ ) relative to healthy controls (median, 100.0; IQR, $16.5 \mathrm{~cm}$ ). MUAC was also lower in HIV mono-infected ART-naive (median, 24.5; IQR, $3.0 \mathrm{~cm} ; P<0.05)$, HIV mono-infected ART-experienced (median, 24.0; IQR, $2.0 \mathrm{~cm} ; P<0.05$ ), HCV monoinfected (median, 26.0; IQR, $3.0 \mathrm{~cm} ; P<0.05$ ), and uninfected (median, 25.0; IQR, $4.0 \mathrm{~cm} ; P<0.05$ ) IDUs compared to healthy controls (median, 29.0; IQR, $7.0 \mathrm{~cm}$ ).

Heroin was the most prevalent injection drug reported among the injection drug using study groups varying from 76.9\% in HIV and HCV co-infected ART-experienced group to $100 \%$ in HIV and HCV co-infected ART-naive and uninfected individuals. Injection of cocaine was also reported in HIV mono-infected ART-experienced (11.8\%) and -naive (6.3\%), HCV mono-infected (11.1\%), and HIV and HCV co-infected ART-experienced (15.4\%) individuals. Injection of both heroin and cocaine was only reported in HIV and HCV co-infected ART-experienced (7.7\%), HCV mono-infected (2.8\%), HIV mono-infected ART-experienced (2.9\%) individuals. Diazepam injection was present in HCV mono-infected (11.1\%), HIV monoinfected ART-experienced (5.9\%) and -naive (12.5\%) individuals while flunitrazepam was found in HIV and HCV co-infected ART-experienced (7.7\%) and HCV monoinfected (5.6\%) individuals.

Non-injection substance consumption was also reported among IDUs. Alcohol, cigarette, bhang and cocktail were the most frequent non-injection substances reported, ranging from $13.9 \%$ in the $\mathrm{HCV}$ mono-infected group to $100.0 \%$ in HIV and HCV co-infected ART-naive individuals. Other non-injection drugs recorded included khat, analgesics, flunitrazepam and brown sugar.

Clinical evaluation indicated that $\mathrm{CD}^{4+} \mathrm{T}$ cell counts were significantly different across the study groups $(P<0.0001)$. Post-hoc analysis showed that the $\mathrm{CD}^{4+}$ $\mathrm{T}$ cell counts were reduced in HIV mono-infected ARTexperienced IDUs (median, 362; IQR, 503/ $\mu$ l) compared to healthy controls (median, 781; IQR, 487/ $\mu \mathrm{l} ; P<0.05$ ). In addition, HIV mono-infected ART-experienced (median, 362; IQR, 503/ $\mu \mathrm{l} ; P<0.001$ ) and -naive (median, 387; IQR, $347 / \mu \mathrm{l} ; P<0.001)$ IDUs presented with lower $\mathrm{CD}^{4+} \mathrm{T}$ cell counts compared to uninfected IDUs (median, 985; IQR, $436 / \mu \mathrm{l})$. Likewise, the $\mathrm{CD}^{4+} \mathrm{T}$ cell counts were lower in HIV and HCV co-infected ART-experienced IDUs (median, 473; IQR, 256/ $\mu \mathrm{l}$ ) compared to HCV mono-infected IDUs (median, 946; IQR, 659/ $\mu \mathrm{l} ; P<0.05$ ). $\mathrm{CD}^{4+} \mathrm{T}$ cell counts were also lower in HIV mono-infected ART-experienced (median, 362; IQR, 503/ $\mu \mathrm{l} ; P<0.001$ ) and -naive (median, 387; IQR, $347 / \mu \mathrm{l} ; P<0.001)$ IDUs in comparison to HCV mono-infected IDUs. However, HIV RNA copies were similar across all the HIV infected study groups $(P=0.557)$.

\section{Adiponectin levels}

Serum adiponectin levels were significantly different across the study groups $(P<0.0001$; Fig. 1). Post-hoc between-group comparisons indicated that adiponectin levels were lower in HIV mono-infected ART-naive IDUs (median, 17.5; IQR, $12.6 \mathrm{ng} / \mathrm{ml}$ ) compared to uninfected IDUs (median, 21.9; IQR, $5.8 \mathrm{ng} / \mathrm{ml} ; P<0.05$ ), and healthy controls (median, 22.0; IQR, $12.5 \mathrm{ng} / \mathrm{ml} ; P<0.05$ ). In contrast, adiponectin levels were higher in HCV monoinfected (median, 23.7; IQR, $4.5 \mathrm{ng} / \mathrm{ml}$ ) compared to HIV

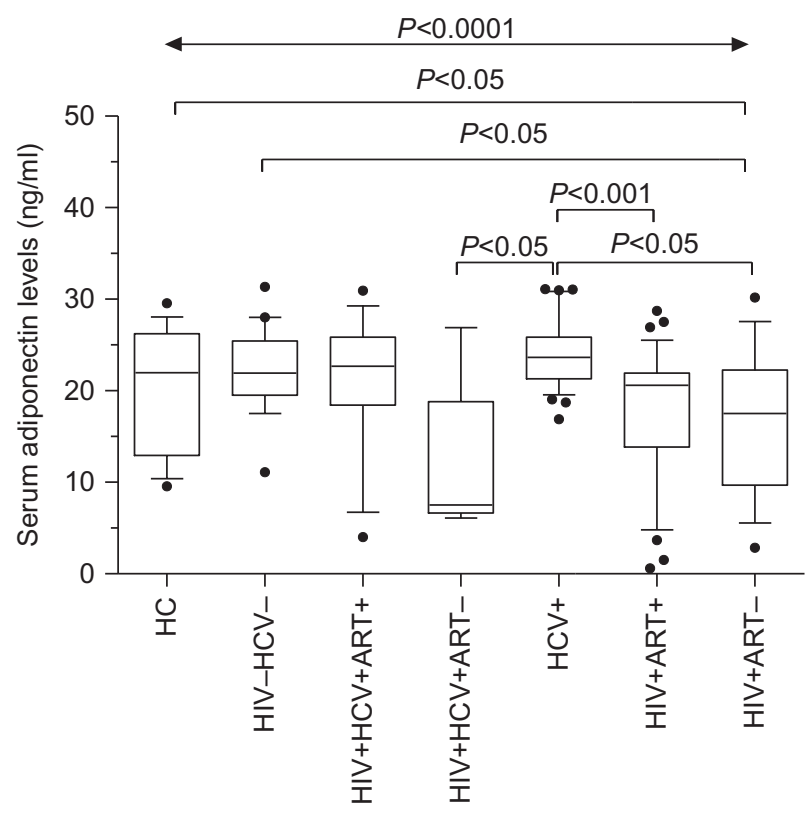

Figure 1

Serum adiponectin levels in the study participants. Box plots showing serum adiponectin levels $(\mathrm{ng} / \mathrm{ml})$ in human immunodeficiency virus (HIV) and hepatitis $\mathrm{C}$ virus (HCV) co-infected anti-retroviral treatment (ART)experienced injection drug users (IDUs) (HIV + HCV + ART +; $n=13) ; \mathrm{HIV}$ and HCV co-infected anti-retroviral treatment-naive IDUs (HIV + HCV + ART - ; $n=5$ ); HCV mono-infected IDUs (HIV-HCV +; $n=36$ ); HIV monoinfected anti-retroviral treatment-experienced IDUs (HIV + HCV - ART+; $n=34$ ); HIV mono-infected anti-retroviral treatment-naive IDUs (HIV+ HCV - ART $-; n=16)$; uninfected IDUs (HIV - HCV $-; n=19$ ), and healthy controls (HC; $n=16)$ from Mombasa, Kenya. Data are presented as box plots, where the box represents the interquartile range, the line through the box represents the median, whiskers indicate the 10th and 90th percentiles, and the closed circles represent outliers. Statistical analysis was conducted using the Kruskal-Wallis test for across group comparison followed by Dunn's correction for multiple comparisons. 
and HCV co-infected ART-naive (median, 7.5; IQR, $12.2 \mathrm{ng} / \mathrm{ml} ; P<0.05)$; and HIV mono-infected ART-naive (median, 17.5; IQR, $12.6 \mathrm{ng} / \mathrm{ml} ; P<0.001$ ) and -experienced (median, 20.6; IQR, $8.1 \mathrm{ng} / \mathrm{ml} ; P<0.001$ ) IDUs.

\section{Associations of adiponectin with anthropometric markers}

The relationship of adiponectin with anthropometric markers in the injection drug using study groups is shown in Table 2 . Adiponectin levels correlated positively with body weight ( $\rho=0.687 ; P=0.003)$ and BMI $(\rho=0.598$; $P=0.014$ ) in HIV mono-infected ART-naive IDUs. In addition, adiponectin levels correlated inversely with waist $(\rho=-0.626 ; P<0.0001)$ and hip $(\rho=-0.561$; $P=0.001)$ circumferences, and positively with bust-to-waist ratio $(\rho=0.561 ; P=0.001)$ in HIV mono-infected ARTexperienced individuals. Furthermore, adiponectin levels were directly associated with waist girth $(\rho=0.375$; $P=0.024)$ in HCV mono-infected individuals. Adiponectin levels were also indirectly associated with waist-to-hip ratio $(\rho=-0.872 ; P=0.048)$ in HIV and HCV co-infected ART-naive individuals.

\section{Discussion}

In this study, we examined the relationship between circulating adiponectin levels and anthropometric markers of adiposity in HIV and HCV mono- and co-infected

Table 2 Correlations of adiponectin with anthropometric indices.

\begin{tabular}{|c|c|c|}
\hline \multirow[b]{2}{*}{ Study group } & \multicolumn{2}{|c|}{ Serum adiponectin levels $(\mathrm{ng} / \mathrm{ml})$} \\
\hline & Rho $(\rho)$ & $P$ \\
\hline \multicolumn{3}{|l|}{$\mathrm{HIV}+\mathrm{ART}-$} \\
\hline Body weight (kg) & 0.687 & 0.003 \\
\hline BMI $\left(\mathrm{kg} / \mathrm{m}^{2}\right)$ & 0.598 & 0.014 \\
\hline \multicolumn{3}{|l|}{$\mathrm{HIV}+\mathrm{ART}+$} \\
\hline Waist circumference $(\mathrm{cm})$ & -0.626 & $<0.0001$ \\
\hline Hip circumference $(\mathrm{cm})$ & -0.561 & 0.001 \\
\hline Bust-to-waist ratio & 0.561 & 0.001 \\
\hline \multicolumn{3}{|l|}{$\mathrm{HCV}+$} \\
\hline Waist circumference $(\mathrm{cm})$ & 0.375 & 0.024 \\
\hline \multicolumn{3}{|l|}{$\mathrm{HCV}+\mathrm{HIV}+\mathrm{ART}-$} \\
\hline Waist-to-hip ratio & -0.872 & 0.048 \\
\hline
\end{tabular}

Data are presented as Spearman's rank correlation co-efficient rho $(\rho)$ with associated $P$ values. HCV, hepatitis C virus; HIV, human immunodeficiency virus; ART, anti-retroviral treatment; HIV + HCV + ART-, HIV and HCV co-infected ART-naive; HCV +, HCV mono-infected; HIV+ART+, HIV mono-infected ART-experienced; HIV+ART - , HIV mono-infected ART-naive. Data analysis was performed using Spearman's rank correlation test. Significant $P$ values are shown in bold.
IDUs. Previous studies show that circulating adiponectin levels are decreased in HIV treatment-naive and HCV mono-infected and co-infected non-substance users (22, $29,30)$. However, circulating adiponectin levels in Kenyan HIV and HCV mono- and co-infected IDUs has not been reported. We found marked reduction in serum adiponectin in HIV mono-infected anti-retroviral-naive IDUs relative to healthy controls and uninfected IDUs, suggesting suppression of adiponectin production. Our results are, in part, consistent with previous studies showing decreased adiponectin protein and mRNA expression in HIV infected anti-retroviral treatmentnaive patients $(22,31)$. Although the underlying mechanisms suppressing adiponectin release were not examined in the current study, opioid illicit drugs activate HIV receptor expression on human adipocytes promoting infectivity, replication and disease progression $(5,32)$. Therefore, HIV infection in IDUs suppresses adipose tissue function leading to reduced adiponectin release.

An inverse link between serum adiponectin levels and BMI and weight has previously been reported in non-drug using HIV uninfected and infected subjects $(33,34)$. In addition, baseline serum adiponectin concentrations correlated inversely with BMI in drug-addicts on methadone maintenance treatment (35). In the present study, adiponectin concentrations were directly correlated with body weight and BMI in HIV mono-infected antiretroviral treatment-naive individuals, suggesting that malnutrition, a frequent morbidity in HIV positive IDUs $(13,36)$, is associated with hypoadiponectinemia. Furthermore, HIV infection and substance consumption dysregulate total body adiposity leading to decreased release of adiponectin $(37,38,39)$. Thus, serum adiponectin levels are valuable correlates of total body adiposity in HIV infected anti-retroviral treatment-naive IDUs.

The indirect relationship of adiponectin with waist and hip circumferences in HIV mono-infected patients on anti-retroviral treatment indicates visceral and subcutaneous adiposity while the positive correlation with bust-to-hip ratio denotes breast region adiposity. Consistent with previous studies in HIV infected non-substance using patients on anti-retroviral treatment (40), a normal balance of adiponectin with visceral and upper trunk subcutaneous adiposity may result from anti-retroviral treatment in HIV infected IDUs. Likewise, our results are, in part, consistent with previous studies showing inverse associations of adiponectin with visceral adipose tissue, isolated central fat accumulation and non-lipodystrophic state in HIV patients on combinational anti-retroviral treatment $(21,41,42,43)$, as well as an indirect link

This work is licensed under a Creative Commons Attribution-NonCommercial 4.0 International License. 
between high molecular weight to total adiponectin ratio and waist circumference in HIV infected women on highly active anti-retroviral treatment (44). Our results also corroborate previous studies showing positive associations between baseline adiponectin and waist circumference in overweight and obese drug addicts (35). We suggest that both injection and non-injection substances alter visceral and subcutaneous adipose tissue redistribution in HIV infected IDUs on anti-retroviral treatment. For instance, a mixed phenotype of HIV-positive patients comprising illicit drug and alcohol use, and anti-retroviral treatmentexperienced individuals exhibit lower and upper trunk adipose depots (45), chronic cannabis smoking is associated with lower abdominal subcutaneous adiposity (46), cigarette smoking is associated with higher visceral adiposity (47), while cathinone, the active compound in khat, induces lipolysis in vivo and inhibit free fatty release in isolated adipocytes (48). Although NRTIs induce increased development of lipodystrophic syndromes in HIV patients $(49,50)$, none of our patients exhibited features of lipodystrophy. Therefore, serum adiponectin is a useful surrogate indicator of altered visceral and subcutaneous adiposity in IDUs on anti-retroviral treatment.

The inverse associations between adiponectin and waist-to-hip ratio in HIV and HCV co-infected antiretroviral treatment-naive IDUs suggests central adiposity, while the positive correlations of adiponectin with waist girth in HCV mono-infected IDUs reflects visceral adiposity. This result, is in part, similar to previous studies showing associations between waist-to-hip ratio and central fat in HIV patients comprising 40\% HCV and IDUs (51). Also, these findings parallel previous studies showing indirect associations between adiponectin levels and waist-to-hip ratio in HIV patients in whom $\sim 67 \%$ were co-infected with HIV and HCV (52). In addition, the positive associations of waist perimeter and adiponectin, are partly, similar to previous studies showing increased fatty liver in individuals with higher waist girth previously observed among HCV infected non-alcoholic patients (53). Abnormal central fat accumulation is a manifestation of HIV-associated lypodystrophy in patients on antiretroviral treatment $(49,50)$. However, central and visceral adiposity in HIV and HCV co-infected treatment-naive and HCV mono-infected IDUs indicates alterations in lipid metabolism. This premise is supported by previous studies in HIV and HCV co-infected patients showing increased risk of having dyslipidaemias in carriers of mitochondrial DNA haplotypes and adiponectin genotypes that are associated with low serum adiponectin levels $(54,55)$. Altogether, adiponectin is a useful marker of central and visceral fat in IDUs presenting with $\mathrm{HCV}$ mono-infection or HIV and HCV co-infections.

The main limitation of this study was the self-reported non-injection substance consumption. In addition, fewer female study participants with HCV and ethnicity could limit evaluation of serum adiponectin with outcomes based on previous studies showing variations in serum adiponectin levels with ethnicity and gender $(56,57)$. However, these factors should not limit interpretation of our results as the major pathogenic outcomes of HIV and HCV are similar across gender and ethnicity. The effect of poly-substance consumption on adiponectin production and adiposity is difficult to assess, as different substances have different effects on the adipose tissue. A prospective design incorporating lipid and toxicological analyses, and other measures of adiposity and clinical staging of HIV and HCV will provide additional insights into the influence of the interaction between HIV and HCV co-infection, injection and noninjection drug use and ART on circulating adiponectin levels. This could not be elucidated in this study owing to the cross-sectional design. In this study, anthropometric measures were taken and correlated with adiponectin levels, even though the clinical utility of some of these measures, especially BMI may be reduced in individuals presenting with lipodystrophy that is common in ART-naive and -experienced HIV infected subjects $(21,58)$.

In summary, our study shows that circulating total adiponectin levels are suppressed in HIV mono-infected anti-retroviral treatment-naive IDUs. Importantly, adiponectin levels are associated with anthropometric measures of adiposity indicating its utility in assessing and monitoring the nutritional profiles of HIV and HCV mono- and co-infected IDUs. This study underscores the complex nature of the interactions of HCV, HIV, ART and injection drug use on adipose tissue and the possible development of metabolic perturbations.

\section{Declaration of interest}

The authors declare that there is no conflict of interest that could be perceived as prejudicing the impartiality of the research reported.

\section{Funding}

This work was, in part, supported by the Kenya National Commission for Science, Technology and Innovation (NCST/5/003/065).

\section{Author contribution statement}

$T$ Were and $V$ Budambula designed, executed, and coordinated the study. M K Webale, F O Musumba, J O Wesongah, and A A Ahmed were involved in the study design and experimental analyses. E M Ndombi and T Were 
performed statistical analyses and co-drafted the manuscript. E Mibei and $\mathrm{R}$ Lihana critically revised the manuscript. All authors have read and approved the final manuscript.

\section{Acknowledgements}

We thank all the study participants and staff and management of Bomu Hospital for their assistance during the study.

\section{References}

1 United Nations Office on Drugs and Crime. World Drug Report 2014, sales no. E.14.XI.7. Vienna, Austria: United Nations, 2014. (available at: https://www.unodc.org/documents/wdr2014/World_Drug_Report_ 2014_web.pdf)

2 Mathers BM, Degenhardt L, Phillips B, Wiessing L, Hickman M, Strathdee SA, Wodak A, Panda S, Tyndall M, Toufik A et al. Global epidemiology of injecting drug use and HIV among people who inject drugs: a systematic review. Lancet 2008372 1733-1745. (doi:10.1016/ S0140-6736(08)61311-2)

3 Nelson PK, Mathers BM, Cowie B, Hagan H, Des Jarlais D, Horyniak D \& Degenhardt L. Global epidemiology of hepatitis B and hepatitis C in people who inject drugs: results of systematic reviews. Lancet 2011378 571-583. (doi:10.1016/S0140-6736(11)61097-0)

4 Soriano V, Barreiro P \& Sherman KE. The changing epidemiology of liver disease in HIV patients. AIDS Reviews 201315 25-31.

5 Edelman EJ, Cheng DM, Krupitsky EM, Bridden C, Quinn E, Walley AY, Lioznov DA, Blokhina E, Zvartau E \& Samet JH. Heroin use and HIV disease progression: results from a pilot study of a Russian cohort. AIDS and Behavior 201419 1089-1097. (doi:10.1007/ s10461-014-0948-z)

6 Zhou Y, Sun L, Wang X, Zhou L, Li J, Liu M, Wang F, Peng J, Gui X, Zhao $\mathrm{H}$ et al. Heroin use promotes HCV infection and dysregulates HCV-related circulating microRNAs. Journal of Neuroimmune Pharmacology 201510 102-110. (doi:10.1007/s11481-014-9577-6)

7 Leclercq P, Goujard C, Duracinsky M, Allaert F, L'Henaff M, Hellet M, Meunier JP, Carret S, Thevenon J, Ngo Van P et al. High prevalence and impact on the quality of life of facial lipoatrophy and other abnormalities in fat tissue distribution in HIV-infected patients treated with antiretroviral therapy. AIDS Research and Human Retroviruses 2012 29 761-768. (doi:10.1089/aid.2012.0214)

8 Palios J, Kadoglou NP \& Lampropoulos S. The pathophysiology of HIV-/HAART-related metabolic syndrome leading to cardiovascular disorders: the emerging role of adipokines. Experimental Diabetes Research 20122012 103063. (doi:10.1155/2012/103063)

9 Loko MA, Bani-Sadr F, Winnock M, Lacombe K, Carrieri P, Neau D, Morlat P, Serfaty L, Dabis F \& Salmon D. Impact of HAART exposure and associated lipodystrophy on advanced liver fibrosis in HIV/HCVcoinfected patients. Journal of Viral Hepatitis 201118 e307-e314. (doi:10.1111/j.1365-2893.2010.01417.x)

10 Syed GH, Amako Y \& Siddiqui A. Hepatitis C virus hijacks host lipid metabolism. Trends in Endocrinology and Metabolism 200921 33-40. (doi:10.1016/j.tem.2009.07.005)

11 Barreira TV, Broyles ST, Gupta AK \& Katzmarzyk PT. Relationship of anthropometric indices to abdominal and total body fat in youth: sex and race differences. Obesity 201422 1345-1350. (doi:10.1002/oby. 20714)

12 Jaswant S \& Nitish M. Use of upper-arm anthropometry as measure of body-composition and nutritional assessment in children and adolescents (6-20 years) of Assam, Northeast India. Ethiopian Journal of Health Sciences 201424 243-252. (doi:10.4314/ejhs.v24i3.8)

13 Tang AM, Bhatnagar T, Ramachandran R, Dong K, Skinner S, Kumar MS \& Wanke CA. Malnutrition in a population of HIV-positive and HIV-negative drug users living in Chennai, South India. Drug and
Alcohol Dependence 2011118 73-77. (doi:10.1016/j.drugalcdep.2011. 02.020)

14 Tang AM, Sheehan HB, Jordan MR, Duong DV, Terrin N, Dong K, Lien TT, Trung NV, Wanke CA \& Hien ND. Predictors of weight change in male HIV-positive injection drug users initiating antiretroviral therapy in Hanoi, Vietnam. AIDS Research and Treatment 20112011 890308. (doi:10.1155/2011/890308)

15 Garg A. Adipose tissue dysfunction in obesity and lipodystrophy. Clinical Cornerstone 20068 (Suppl 4) S7-S13. (doi:10.1016/S10983597(06)80039-6)

16 Carbone F, La Rocca C \& Matarese G. Immunological functions of leptin and adiponectin. Biochimie 201294 2082-2088. (doi:10.1016/j. biochi.2012.05.018)

17 Housova J, Wilczek H, Haluzik MM, Kremen J, Krizova J \& Haluzik M. Adipocyte-derived hormones in heroin addicts: the influence of methadone maintenance treatment. Physiological Research 2005 54 73-78.

18 Iwashima Y, Katsuya T, Ishikawa K, Kida I, Ohishi M, Horio T, Ouchi N, Ohashi K, Kihara S, Funahashi T et al. Association of hypoadiponectinemia with smoking habit in men. Hypertension 200545 1094-1100. (doi:10.1161/01.HYP.0000169444.05588.4c)

19 Levandowski ML, Viola TW, Tractenberg SG, Teixeira AL, Brietzke E, Bauer ME \& Grassi-Oliveira R. Adipokines during early abstinence of crack cocaine in dependent women reporting childhood maltreatment. Psychiatry Research 2013210 536-540. (doi:10.1016/j. psychres.2013.07.007)

20 Buechler C, Schaffler A, Johann M, Neumeier M, Kohl P, Weiss T, Wodarz N, Kiefer P \& Hellerbrand C. Elevated adiponectin serum levels in patients with chronic alcohol abuse rapidly decline during alcohol withdrawal. Journal of Gastroenterology and Hepatology 200924 558-563. (doi:10.1111/j.1440-1746.2008.05693.x)

21 Addy CL, Gavrila A, Tsiodras S, Brodovicz K, Karchmer AW \& Mantzoros CS. Hypoadiponectinemia is associated with insulin resistance, hypertriglyceridemia, and fat redistribution in human immunodeficiency virus-infected patients treated with highly active antiretroviral therapy. Journal of Clinical Endocrinology and Metabolism 200388 627-636. (doi:10.1210/jc.2002-020795)

22 Das S, Shahmanesh M, Stolinski M, Shojaee-Moradie F, Jefferson W, Jackson NC, Cobbold M, Nightingale P \& Umpleby AM. In treatmentnaive and antiretroviral-treated subjects with HIV, reduced plasma adiponectin is associated with a reduced fractional clearance rate of VLDL, IDL and LDL apolipoprotein B-100. Diabetologia 200649 538-542. (doi:10.1007/s00125-005-0085-3)

23 Sutinen J, Korsheninnikova E, Funahashi T, Matsuzawa Y, Nyman T \& Yki-Jarvinen H. Circulating concentration of adiponectin and its expression in subcutaneous adipose tissue in patients with highly active antiretroviral therapy-associated lipodystrophy. Journal of Clinical Endocrinology and Metabolism 200388 1907-1910. (doi:10.1210/ jc.2002-021922)

24 Palmer C, Hampartzoumian T, Lloyd A \& Zekry A. A novel role for adiponectin in regulating the immune responses in chronic hepatitis $\mathrm{C}$ virus infection. Hepatology 200848 374-384. (doi:10.1002/hep.22387)

25 Tang AM, Forrester JE, Spiegelman D, Flanigan T, Dobs A, Skinner S \& Wanke C. Heavy injection drug use is associated with lower percent body fat in a multi-ethnic cohort of HIV-positive and HIV-negative drug users from three U.S. cities. American Journal of Drug and Alcohol Abuse 201036 78-86. (doi:10.3109/00952990903544851)

26 Were T, Wesongah JO, Munde E, Ouma C, Kahiga TM, OngechaOwuor F, Kiarie JN, Ahmed AA, Makokha EP \& Budambula V. Clinical chemistry profiles in injection heroin users from Coastal Region, Kenya. BMC Clinical Pathology 2014 14 32. (doi:10.1186/1472-6890-14-32)

27 Ministry of Health; National AIDS and STI Control Program (NASCOP). Guidelines on Use of Antiretroviral Drugs for Treating and Preventing HIV Infection: A rapid advice. Nairobi, Kenya: NASCOP, 2014.

28 WHO. WHO STEPS Surveilance Part 3: Training and Practical Guides Geneva, Switzerland: WHO, 2008. (available at: http://www.who.int/ chp/steps/Part3.pdf) 
29 Korah TE, El-Sayed S, Elshafie MK, Hammoda GE \& Safan MA. Significance of serum leptin and adiponectin levels in Egyptian patients with chronic hepatitis $\mathrm{C}$ virus associated hepatic steatosis and fibrosis. World Journal of Hepatology 2013 5 74-81. (doi:10.4254/wjh.v5.i2.74)

30 Veloso S, Escote X, Ceperuelo-Mallafre V, Lopez-Dupla M, Peraire J, Vilades C, Domingo P, Castro A, Olona M, Sirvent JJ et al. Leptin and adiponectin, but not IL18, are related with insulin resistance in treated HIV-1-infected patients with lipodystrophy. Cytokine 201258 253-260. (doi:10.1016/j.cyto.2012.01.013)

31 Giralt M, Domingo P, Guallar JP, Rodriguez de la Concepcion ML, Alegre M, Domingo JC \& Villarroya F. HIV-1 infection alters gene expression in adipose tissue, which contributes to HIV- 1/HAARTassociated lipodystrophy. Antiviral Therapy 200611 729-740.

32 Hazan U, Romero IA, Cancello R, Valente S, Perrin V, Mariot V, Dumonceaux J, Gerhardt CC, Strosberg AD, Couraud PO et al. Human adipose cells express CD4, CXCR4, and CCR5 receptors: a new target cell type for the immunodeficiency virus-1? FASEB Journal 200216 1254-1256. (doi:10.1096/fj.01-0947fje)

33 Matsubara M, Maruoka S \& Katayose S. Inverse relationship between plasma adiponectin and leptin concentrations in normal-weight and obese women. European Journal of Endocrinology 2002147 173-180. (doi:10.1530/eje.0.1470173)

34 Tong Q, Sankale JL, Hadigan CM, Tan G, Rosenberg ES, Kanki PJ, Grinspoon SK \& Hotamisligil GS. Regulation of adiponectin in human immunodeficiency virus-infected patients: relationship to body composition and metabolic indices. Journal of Clinical Endocrinology and Metabolism 200388 1559-1564. (doi:10.1210/jc. 2002-021600)

35 Montazerifar F, Karajibani M, Lashkaripour K \& Yousefi M. Evaluation of serum adiponectin concentrations among drug abusers on methadone maintenance treatment. International Journal of High Risk Behaviors \& Addiction 20142 117-121. (doi:10.5812/ijhrba.14021)

36 Forrester JE, Tucker KL \& Gorbach SL. Dietary intake and body mass index in HIV-positive and HIV-negative drug abusers of Hispanic ethnicity. Public Health Nutrition 20047 863-870. (doi:10.1079/ PHN2004617)

37 Flechtner-Mors M, George SN, Oeztuerk S, Haenle MM, Koenig W, Imhof A, Boehm BO, Graeter T, Mason RA, Kratzer W et al. Association of adiponectin with hepatic steatosis: a study of 1,349 subjects in a random population sample. BMC Research Notes 20147207. (doi:10.1186/1756-0500-7-207)

38 Sankale JL, Tong Q, Hadigan CM, Tan G, Grinspoon SK, Kanki PJ \& Hotamisligil GS. Regulation of adiponectin in adipocytes upon exposure to HIV-1. HIV Medicine 20067 268-274. (doi:10.1111/j.14681293.2006.00372.x)

39 Teixeira D, Pestana D, Faria A, Calhau C, Azevedo I \& Monteiro R. Modulation of adipocyte biology by $\delta(9)$-tetrahydrocannabinol. Obesity 201018 2077-2085. (doi:10.1038/oby.2010.100)

40 Kosmiski L, Kuritzkes D, Lichtenstein K \& Eckel R. Adipocyte-derived hormone levels in HIV lipodystrophy. Antiviral Therapy 2003 8 9-15.

41 Brown TT, Chen Y, Currier JS, Ribaudo HJ, Rothenberg J, Dube MP, Murphy R, Stein JH \& McComsey GA. Body composition, soluble markers of inflammation, and bone mineral density in antiretroviral therapy-naive HIV-1-infected individuals. Journal of Acquired Immune Deficiency Syndromes 201363 323-330. (doi:10.1097/QAI. Ob013e318295eb1d)

42 Freitas P, Carvalho D, Santos AC, Madureira AJ, Martinez E, Pereira J, Sarmento A \& Medina JL. Adipokines, hormones related to body composition, and insulin resistance in HIV fat redistribution syndrome. BMC Infectious Diseases 201414 347. (doi:10.1186/ 1471-2334-14-347)

43 Samaras K, Wand H, Law M, Emery S, Cooper D \& Carr A. Prevalence of metabolic syndrome in HIV-infected patients receiving highly active antiretroviral therapy using International Diabetes Foundation and
Adult Treatment Panel III criteria: associations with insulin resistance, disturbed body fat compartmentalization, elevated C-reactive protein, and hypoadiponectinemia. Diabetes Care 200730 113-119. (doi:10.2337/dc06-1075)

44 Omar F, Dave JA, King JA, Levitt NS \& Pillay TS. High Molecular Weight (HMW): total adiponectin ratio is low in HIV-infected women receiving protease inhibitors. BMC Clinical Pathology 201514 46. (doi:10.1186/ 1472-6890-14-46)

45 Grunfeld C, Rimland D, Gibert CL, Powderly WG, Sidney S, Shlipak MG, Bacchetti P, Scherzer R, Haffner S \& Heymsfield SB. Association of upper trunk and visceral adipose tissue volume with insulin resistance in control and HIV-infected subjects in the FRAM study. Journal of Acquired Immune Deficiency Syndromes 200746 283-290. (doi:10.1097/QAI.0b013e31814b94e2)

46 Muniyappa R, Sable S, Ouwerkerk R, Mari A, Gharib AM, Walter M, Courville A, Hall G, Chen KY, Volkow ND et al. Metabolic effects of chronic cannabis smoking. Diabetes Care 201336 2415-2422. (doi:10.2337/dc12-2303)

47 Demerath EW, Rogers NL, Reed D, Lee M, Choh AC, Siervogel RM, Chumlea WC, Towne B \& Czerwinski SA. Significant associations of age, menopausal status and lifestyle factors with visceral adiposity in African-American and European-American women. Annals of Human Biology 201038 247-256. (doi:10.3109/03014460. 2010.524893)

48 Nencini P. Cathinone, active principle of the khat leaf: its effects on in vivo and in vitro lipolysis. Pharmacological Research Communications 198012 855-861. (doi:10.1016/S0031-6989 (80)80048-8)

49 Arama V, Munteanu DI, Streinu Cercel A, Ion DA, Mihailescu R, Tiliscan C, Tudor AM \& Arama SS. Lipodystrophy syndrome in HIV treatment-multiexperienced patients: implication of resistin. Journal of Endocrinological Investigation 201437 533-539. (doi:10.1007/s40618014-0057-x)

50 Estrada V, Martinez-Larrad MT, Gonzalez-Sanchez JL, de Villar NG, Zabena C, Fernandez C \& Serrano-Rios M. Lipodystrophy and metabolic syndrome in HIV-infected patients treated with antiretroviral therapy. Metabolism 200655 940-945. (doi:10.1016/j.metabol. 2006.02.024)

51 Araujo S, Banon S, Machuca I, Moreno A, Perez-Elias MJ \& Casado JL. Prevalence of insulin resistance and risk of diabetes mellitus in HIV-infected patients receiving current antiretroviral drugs. European Journal of Endocrinology 2014171 545-554. (doi:10.1530/ EJE-14-0337)

52 Parfieniuk-Kowerda A, Czaban SL, Grzeszczuk A, Jaroszewicz J \& Flisiak R. Assessment of serum IGF-1 and adipokines related to metabolic dysfunction in HIV-infected adults. Cytokine 201364 97-102. (doi:10.1016/j.cyto.2013.07.018)

53 Gonzalez-Reimers E, Castellano-Higuera A, Aleman-Valls R, AlvarezArguelles H, de la Vega-Prieto MJ, Abreu-Gonzalez P, Lopez-Prieto J, Santolaria-Fernandez F \& Valladares-Parrilla F. Relation between body fat and liver fat accumulation and cytokine pattern in non-alcoholic patients with chronic HCV infection. Annals of Nutrition \& Metabolism 200955 351-357. (doi:10.1159/000252351)

54 Micheloud D, Berenguer J, Guzman-Fulgencio M, Campos Y, GarciaAlvarez M, Catalan P, Cosin J, Miralles P, Lopez JC \& Resino S. European mitochondrial DNA haplogroups and metabolic disorders in HIV/HCVcoinfected patients on highly active antiretroviral therapy. Journal of Acquired Immune Deficiency Syndromes 201158 371-378. (doi:10.1097/ QAI.0b013e31822d2629)

55 Pineda-Tenor D, Berenguer J, Garcia-Broncano P, Jimenez-Sousa MA, Fernandez-Rodriguez A, Diez C, Garcia-Alvarez M, Carrero A, Catalan P, Aldamiz-Echevarria $\mathrm{T}$ et al. Association of adiponectin (ADIPOQ) rs2241766 polymorphism and dyslipidemia in HIV/HCV-coinfected patients. European Journal of Clinical Investigation $2014 \mathbf{4 4} 453-462$. (doi:10.1111/eci.12250) 
56 Schutte AE, Huisman HW, Schutte R, Malan L, van Rooyen JM, Malan NT \& Schwarz PE. Differences and similarities regarding adiponectin investigated in African and Caucasian women. European Journal of Endocrinology 2007157 181-188. (doi:10.1530/EJE-07-0044)

57 Sobngwi E, Effoe V, Boudou P, Njamen D, Gautier J-F \& Mbanya J-C. Waist circumference does not predict circulating adiponectin levels in
sub-Saharan women. Cardiovascular Diabetology 2007631.

(doi:10.1186/1475-2840-6-31)

58 Vigouroux C, Maachi M, Nguyen TH, Coussieu C, Gharakhanian S, Funahashi T, Matsuzawa Y, Shimomura I, Rozenbaum W, Capeau J et al. Serum adipocytokines are related to lipodystrophy and metabolic disorders in HIV-infected men under antiretroviral therapy. AIDS 2003 17 1503-1511. (doi:10.1097/00002030-200307040-00011)

Received in final form 28 July 2015

Accepted 25 August 2015 